\title{
Conduction-dominated heat transport of the annual temperature signal in soil
}

\author{
Jason E. Smerdon, ${ }^{1}$ Henry N. Pollack, ${ }^{2}$ John W. Enz, ${ }^{3}$ and Matthew J. Lewis ${ }^{4}$ \\ Received 18 December 2002; revised 22 May 2003; accepted 13 June 2003; published 17 September 2003.
}

[1] Conductive heat transport of temperature signals into the subsurface is a central assumption of ground surface temperature (GST) reconstructions derived from presentday temperatures in deep boreholes. Here we test this assumption and its implications for annual relationships between GST and surface air temperature (SAT) by analyzing two decades of shallow soil temperature $(0.01-11.7 \mathrm{~m})$ and SAT time series measured at Fargo, North Dakota. We spectrally decompose each of these temperature time series to determine the amplitude and phase of the annual signal at each depth. Conductive heat transport of a harmonic temperature signal in a homogeneous medium is characterized theoretically by exponential amplitude attenuation and linear phase shift with depth. We show that transport of the annual signal in the soil at Fargo follows these theoretical characterizations of conduction closely: the depth dependence of both the natural logarithm of the amplitude and the phase shift are highly linear. Interval wave velocities and thermal diffusivities calculated as functions of depth suggest a diffusivity gradient in the upper meter of the soil. We estimate the annual signal at the ground surface by extrapolating amplitude and phase shift regression lines upward to the surface. We compare this estimate of the annual signal at the ground surface to the annual signal contained in the SAT and show the ground surface signal to be attenuated $\sim 20 \%$ and negligibly phase shifted relative to the SAT. INDEX TERMS: 1645 Global Change: Solid Earth; 1875 Hydrology: Unsaturated zone; 3322 Meteorology and Atmospheric Dynamics: Land/atmosphere interactions; 3344 Meteorology and Atmospheric Dynamics: Paleoclimatology; 3367 Meteorology and Atmospheric Dynamics: Theoretical modeling; KEYWORDS: paleoclimate, soil temperature, conductive heat transport

Citation: Smerdon, J. E., H. N. Pollack, J. W. Enz, and M. J. Lewis, Conduction-dominated heat transport of the annual temperature signal in soil, J. Geophys. Res., 108(B9), 2431, doi:10.1029/2002JB002351, 2003.

\section{Introduction}

[2] Reconstructions of ground surface temperature (GST) histories using present-day measurements of temperature in deep boreholes have been used as estimates of climatic change over a large range of spatial and temporal scales (for a review, see Pollack and Huang [2000]). General agreement between GST reconstructions and historical SAT records is well documented [e.g., Huang et al., 2000; Harris and Chapman, 2001; Beltrami, 2002]. Such agreement has given credence to the method of GST reconstruction and to the idea that GST reconstructions reflect the generalized climate history of a region. Virtually all of the common methods of reconstructing and interpreting GST

\footnotetext{
${ }^{1}$ Applied Physics Program, University of Michigan, Ann Arbor, Michigan, USA

${ }^{2}$ Department of Geological Sciences, University of Michigan, Ann Arbor, Michigan, USA.

${ }^{3}$ Department of Soil Science, North Dakota State University, Fargo, North Dakota, USA.

${ }^{4}$ Michigan Center for Theoretical Physics, University of Michigan, Ann Arbor, Michigan, USA.

Copyright 2003 by the American Geophysical Union. 0148-0227/03/2002JB002351\$09.00
}

histories are based on two assumptions: (1) the propagation of temperature signals into the subsurface takes place by thermal conduction; and (2) changes in GST are closely coupled to changes in surface air temperature (SAT), enabling GST reconstructions to be reliable estimates of long-term changes in SAT. This paper addresses principally assumption 1 within the context of the shallow soil but also touches on assumption 2 by providing a quantitative comparison of GST and SAT annual signals.

[3] GST reconstructions represent a filtered version of GST history that is absent of short-period variations such as diurnal or seasonal cycles. These temperature cycles are attenuated relatively quickly within the soil and cannot typically be resolved below 10-m depth, the standard depth at which borehole temperature profiles used for GST reconstructions begin. Nevertheless, long-term temperature variations that propagate to greater subsurface depths are established at or near the ground surface as a result of changes in net energy fluxes occurring over very short timescales. Thus it is important to understand how temperature signals are propagated through the very shallow soil and how various conditions at the ground surface and in the shallow subsurface affect the signal and its downward propagation. Because reconstructions of GST histories 
assume that temperature changes at the ground surface propagate into the subsurface conductively, it is important to specifically verify that conduction is the principal mode of heat transfer within the shallow soil.

[4] A variety of meteorological and agricultural research stations have assembled valuable data comprising time series measurements of shallow soil temperatures and meteorological conditions that have allowed for empirical studies of air and soil temperature relationships [e.g., Baker and Ruschy, 1993; Putnam and Chapman, 1996; Beltrami, 2001; Zhang et al., 2001; Baker and Baker, 2002]. The primary focus of these studies has been to assess how climatically driven processes at or near the ground surface, including air temperature, generate and influence the downward propagating subsurface climate signal. These processes most notably include the effects of snow cover, soil freezing, evapotranspiration, and vegetative changes. Studies of air and soil temperature relationships at specific sites where these processes are known to be active can therefore provide valuable insights into their affects on the subsurface climate signal as well as place constraints on the magnitude of their influence on large-scale GST reconstructions.

[5] Here we test the hypothesis of heat conduction with an analysis of temperature time series measured in the shallow soil for two decades at Fargo, North Dakota. Schmidt et al. [2001] have analyzed the first decade of this data for different purposes using a different methodological approach. We utilize the full two decades of data to compare the downward propagation of the annual signal with predictions from a one-dimensional model of heat conduction in a homogeneous medium. We focus on the annual signal for three reasons: (1) the seasonal variability of temperature at the Earth surface is closely approximated by a simple harmonic function; (2) the annual signal is the dominant signal within the range of depths observed; and (3) the two decades of data at the Fargo site are well suited to tracking the annual signal. We test the propagation behavior of the annual signal against two characterizations of downward conductive heat transport of a harmonic temperature function imposed at the surface of a homogenous medium: (1) exponential attenuation of the amplitude of the harmonic function with depth; and (2) linear phase shift of the harmonic function with depth. Interval wave velocities and thermal diffusivities are calculated from the phase shift of the annual signal to estimate changing thermal properties as a function of depth. We also extrapolate our results to estimate the amplitude and phase of the annual signal at the ground surface.

\section{Data}

[6] We analyze air and soil temperatures measured at the North Dakota State University (NDSU) Microclimate Research Station $\left(46^{\circ} 54^{\prime} \mathrm{N}, 96^{\circ} 48^{\prime} \mathrm{W}\right)$ in Fargo, North Dakota. The site and data acquisition are described by Schmidt et al. [2001]. Here we use approximately two decades of air and soil temperatures from 1 September 1980 to 31 August 1999, a time period of 6939 days. We analyze air temperatures recorded at a height of $1.5 \mathrm{~m}$ and soil temperatures recorded at depths of $0.01,0.05$, $0.1,0.2,0.3,0.4,0.5,0.6,0.8,1.0,1.25,1.5,1.75,2.0$,
Table 1. Gaps Within the North Dakota State University Temperature Record Larger Than 10 Days, the Number of Days in Each Gap, and the Ensemble of Years Used for Average Interpolation of the Gaps

\begin{tabular}{|c|c|c|}
\hline $\begin{array}{c}\text { Time Interval } \\
\text { of Gap }\end{array}$ & $\begin{array}{l}\text { Number of } \\
\text { Missing Days }\end{array}$ & $\begin{array}{c}\text { Years Used for } \\
\text { Average Interpolation }\end{array}$ \\
\hline 1 April to 31 Oct. 1990 & 214 & $1981-1989,1991,1997$ and 1998 \\
\hline 27 Nov. to 31 Dec. 1991 & 35 & $\begin{array}{l}1980-1990,1992-1994 \\
1996 \text { and } 1997\end{array}$ \\
\hline 1 May to 30 Sept. 1992 & 122 & $1981-1989,1991,1997$ and 1998 \\
\hline 23 April to 4 May 1993 & 12 & $1981-1989,1991,1997$ and 1998 \\
\hline 23 June to 7 July 1993 & 15 & $1981-1989,1991,1997$ and 1998 \\
\hline $1-30$ June 1994 & 30 & $1981-1989,1991,1997$ and 1998 \\
\hline 23 Aug. to 2 Sept. 1994 & 11 & $1981-1989,1991,1997$ and 1998 \\
\hline $1-30$ Sept. 1995 & 30 & $1981-1989,1991,1997$ and 1998 \\
\hline 1-30 Nov. 1995 & 30 & $\begin{array}{l}1980-1990,1992-1994 \\
1996 \text { and } 1997\end{array}$ \\
\hline 1-31 May 1996 & 31 & $1981-1989,1991,1997$ and 1998 \\
\hline
\end{tabular}

$2.5,3.0,3.7,4.7,5.7,7.7,9.7$, and $11.7 \mathrm{~m}$. We do not use the soil temperatures recorded at $2.7 \mathrm{~m}$ because of evidence suggesting calibration problems at that depth [Schmidt et al., 2001].

\subsection{Data Aggregation and Interpolation}

[7] Temperatures were measured at each depth every hour. We have aggregated hourly measurements into daily averages. Each time series is missing a total number of 712 days heterogeneously distributed throughout the period of observation, but all gaps occur during the same time intervals at all depths. To facilitate Fourier analysis of the time series, we interpolate through each gap to create records with equal time steps of one day. Of the total missing days in the record, $26 \%$ comprise gaps of 10 days or less that have been filled by linear interpolation. The remaining $74 \%$ of the missing days are concentrated into 10 intervals that range from 11 to 214 days in duration. These gaps were bridged by "average interpolation," after linear interpolation of the shorter gaps was completed. Average interpolation fills each day of a gap with the average daily temperature from an ensemble of years that do not contain the gap. Table 1 displays the time period of each gap longer than 10 days and the ensemble of years used for average interpolation of the gaps.

\subsection{General Climate at Fargo}

[8] Figure 1(top) displays the interpolated SAT time series observed at the NDSU site. During the period of observation, maximum and minimum SAT at Fargo ranged between approximately $-35^{\circ} \mathrm{C}$ and $35^{\circ} \mathrm{C}$; freezing temperatures typically began in October and persisted into April. The mean annual temperature between 1981 and 1998, excluding the largely incomplete years of 1990 and 1992 , was $5.8^{\circ} \mathrm{C}$. Precipitation data measured at the Hector International Airport in Fargo is shown in Figure 2; these data were obtained from the National Climate Data Center. The mean annual rain-equivalent precipitation between 1981 and 1999 was $52.4 \mathrm{~cm}$. During the same period, snowfall typically occurred between the months of October and April and the mean annual snowfall was $123 \mathrm{~cm}$. Clearly, the Fargo site is a good location to evaluate cryogenic effects on subsurface temperatures. 


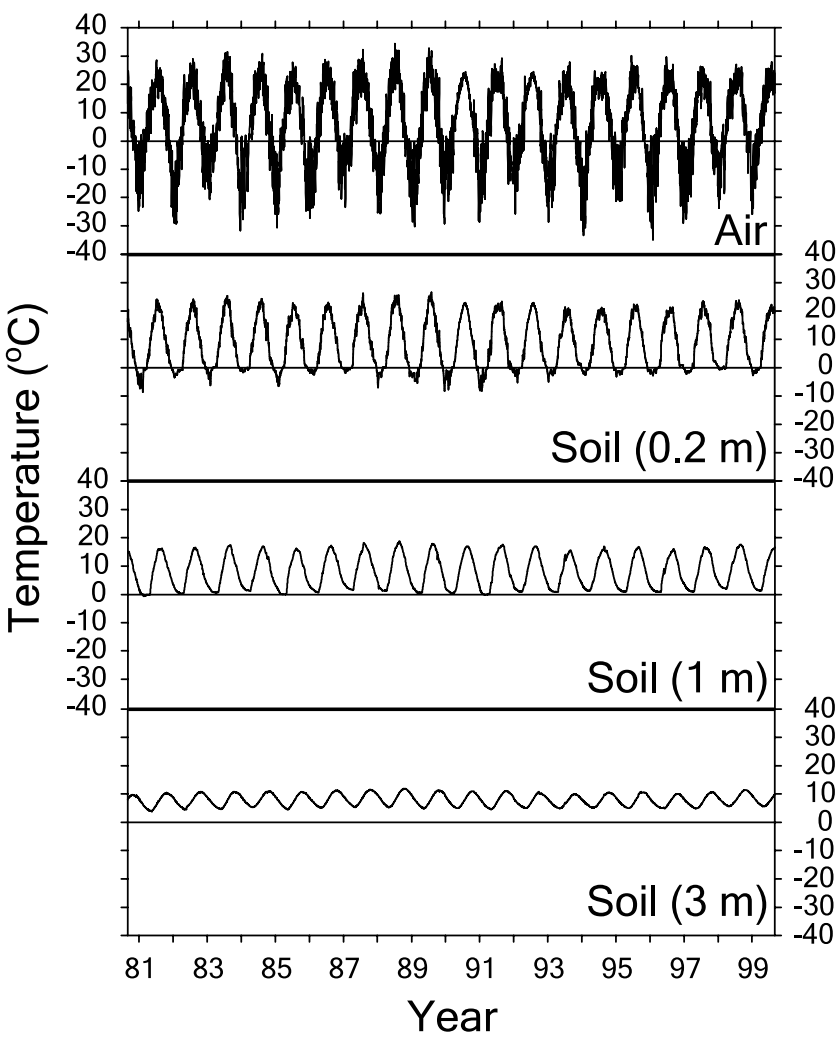

Figure 1. Interpolated SAT time series and soil temperature time series at $0.2,1$ and $3 \mathrm{~m}$ depth.

[9] Three examples of soil temperature time series measured at $0.2,1$ and $3 \mathrm{~m}$ are also shown in Figure 1 (interpolations are included). Visual inspection of the time series reveals several important characteristics of air and soil temperature relationships: (1) high-frequency temperature perturbations present in the SAT are progressively filtered with depth and have almost disappeared at a depth of $1 \mathrm{~m}$; (2) soil freezing is apparent in the 0.2-m measurement but largely absent at $1 \mathrm{~m}$ and below; (3) mean annual soil temperatures are warmer than the mean annual SAT (between 1981 and 1998, excluding 1990 and 1992, they were $8.4^{\circ} \mathrm{C}, 8.0^{\circ} \mathrm{C}$ and $7.9^{\circ} \mathrm{C}$ at $0.2,1$ and $3 \mathrm{~m}$, respectively, and mean SAT was $5.8^{\circ} \mathrm{C}$ ); and (4) the annual signal present

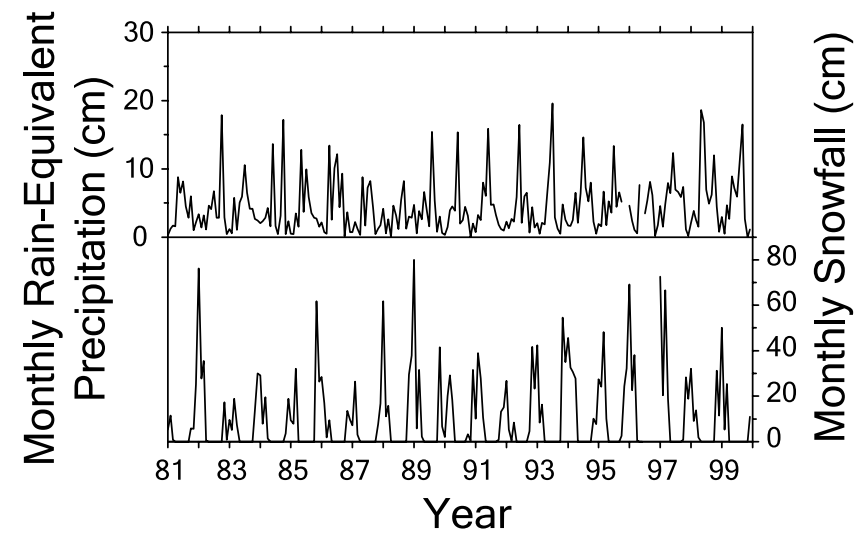

Figure 2. Monthly (top) rain-equivalent precipitation and (bottom) snowfall measured at Fargo, North Dakota. in the soil temperature time series is clearly attenuated and phase shifted relative to the air temperature signal.

\section{Analysis of Soil Temperatures in a Homogeneous Conductive Medium}

\subsection{Amplitude and Phase Tracking}

[10] The characteristics of conductive heat transport are widely explored within the literature (for an extensive discussion, see Carslaw and Jaeger [1959]). Here we utilize the steady state analytic solution of the one-dimensional heat conduction equation for a simple harmonic surface
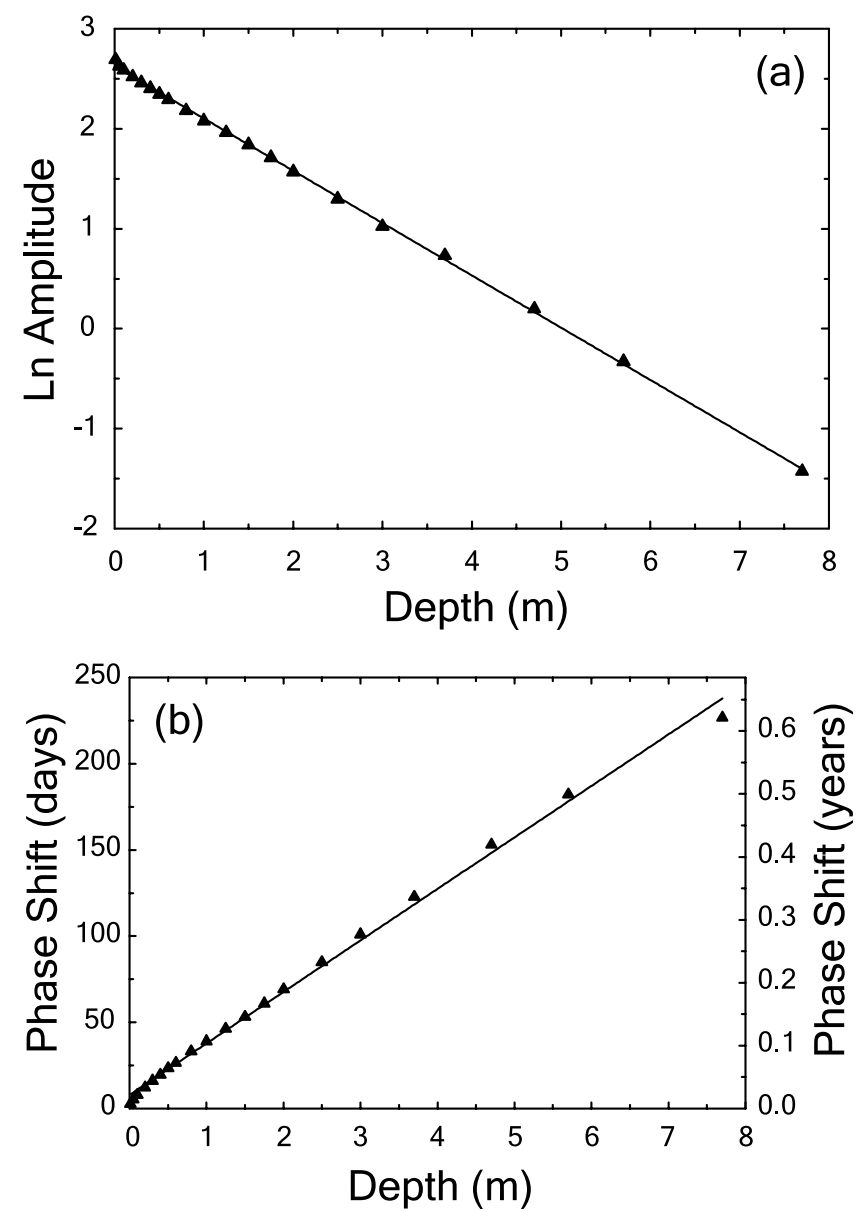

Figure 3. (a) Natural logarithm of the amplitude of the annual signal plotted against depth. Linear regression yields a slope of $-5.23 \pm 0.03 \times 10^{-1} \mathrm{~m}^{-1}$ that is related to the wave vector of the propagating wave and gives an estimate of the mean thermal diffusivity of $3.64 \pm 0.04 \times$ $10^{-7} \mathrm{~m}^{2} \mathrm{~s}^{-1}$. The zero-depth intercept of the line yields an estimate of the amplitude of the annual signal in the ground surface temperature of $13.8 \pm 0.1 \mathrm{~K}$. (b) Phase shift of the annual signal plotted against depth. Linear regression yields a slope of $5.14 \pm 0.07 \times 10^{-1} \mathrm{rad} \mathrm{m}^{-1}$ that is related to the wave vector of the propagating wave and gives an estimate of the average thermal diffusivity of $3.8 \pm 0.1 \times$ $10^{-7} \mathrm{~m}^{2} \mathrm{~s}^{-1}$. The zero-depth intercept of $8 \pm 1$ days is an estimate of the phase shift of the annual signal in the ground surface temperature relative to the SAT (all phases were measured relative to the SAT). 
Table 2. Thermal Diffusivity and Wave Velocity Estimated From Linear Regression of the Natural Logarithm of the Amplitude and Phase Shift of the Annual Signal as Functions of Depth

\begin{tabular}{lccc}
\hline \multicolumn{1}{c}{ Data Source } & Wave Vector (Regression Slope) $k, \mathrm{~m}^{-1}$ & Thermal Diffusivity $k, \mathrm{~m}^{2} \mathrm{~s}^{-1}$ & Wave Velocity $v, \mathrm{~m} \mathrm{yr}$ \\
\hline Natural logarithm & & & \\
$\quad$ amplitude regression & $0.523 \pm 0.003$ & $3.64 \pm 0.04 \times 10^{-7}$ & $12.0 \pm 0.4$ \\
Phase shift regression & $0.514 \pm 0.007$ & $3.8 \pm 0.1 \times 10^{-7}$ & $12.3 \pm 0.1$ \\
Average of regression results & $0.518 \pm 0.007$ & $3.7 \pm 0.1 \times 10^{-7}$ & $12.1 \pm 0.2$ \\
From seasonal and layered & & $3.8 \times 10^{-7}$ & 12.3 \\
$\quad$ analysis of Schmidt et al. $[2001]$ & 0.512 & & \\
\hline
\end{tabular}

temperature signal propagating into a homogeneous halfspace. The downward propagating temperature signal as a function of time $(t)$ and depth $(z)$ is given by Carslaw and Jaeger [1959] as $T(z, t)=A e^{-k z} \cos (\omega t+\epsilon-k z)$, where $A$ and $\omega$ are the amplitude and angular frequency of the harmonic surface temperature signal, respectively, and $\epsilon$ is the initial phase of the surface signal. The solution represents a thermal wave with wave vector $k=(\pi / P \kappa)^{1 / 2}$ and wavelength $\lambda=(4 \pi P \kappa)^{1 / 2}$, where $P$ and $\kappa$ are the period of oscillation and the thermal diffusivity of the conducting medium, respectively. The propagating thermal wave has a constant wave velocity, $v=(4 \pi \kappa / P)^{1 / 2}$.

[11] The amplitude and phase of the annual signal within all of the temperature time series measured at the NDSU site were extracted using Fourier analysis and are plotted versus depth in Figure 3. The power of the annual signal diminishes with depth, and the signal cannot be resolved below the 7.7-m measurement. Consequently, the 9.7- and $11.7-\mathrm{m}$ measurements have not been included in our analyses. The phase at each depth has been taken relative to the phase of the annual signal in the SAT. Regressions of the two data series show that a linear model describes the depth dependence of the natural logarithm of the amplitude and the phase shift with high fidelity; linear regressions yield coefficient of determination $\left(r^{2}\right)$ values of 0.999 and 0.996 , respectively. The slopes of each regression line enable estimates of the wave vector of the propagating wave. In turn, the mean thermal diffusivity of the subsurface and the mean annual wave velocity are calculated from the wave vector estimates. The results of our calculations appear in Table 2.

[12] The calculated average thermal diffusivity of $3.7 \pm$ $0.1 \times 10^{-7} \mathrm{~m}^{2} \mathrm{~s}^{-1}$ is an average over all seasons and all depths. For comparison, we compute the average thermal diffusivity from the seasonal and layered thermal conductivity analysis presented by Schmidt et al. [2001]. Schmidt et al. used the constant values of $1000 \mathrm{~J} \mathrm{~kg}^{-1} \mathrm{~K}^{-1}$ and $2200 \mathrm{~kg} \mathrm{~m}^{-3}$ for specific heat and density, respectively, and performed a best fit model analysis of the Fargo data (1981-1989) to estimate a thermal conductivity range between 0.52 and $1.57 \mathrm{~W} \mathrm{~m}^{-1} \mathrm{~K}^{-1}$ that varies over seasons and depth. The average of all the conductivities given by Schmidt et al. [2001] is $0.84 \mathrm{~W} \mathrm{~m}^{-1} \mathrm{~K}^{-1}$, corresponding to a thermal diffusivity of $3.8 \times 10^{-7} \mathrm{~m}^{2} \mathrm{~s}^{-1}$ (see Table 2).

\subsection{Regression Residuals and Interval Wave Velocities}

[13] The principal conclusion of the regression analysis is that a homogeneous conductive model closely describes the subsurface heat transport regime over all depths observed. There are, however, small details within the regression residuals that give additional insights into the nature of heat transport within the soil. Analyses of the residuals shown in
Figure 4 reveal small but systematic residuals in the upper meter of the soil, suggesting some inhomogeneity in that zone. We also note that the standardized residuals suggest a modest statistical outlier in the phase shift data at the 7.7-m depth. This undoubtedly is a result of the low power associated with the annual signal at that depth. The data
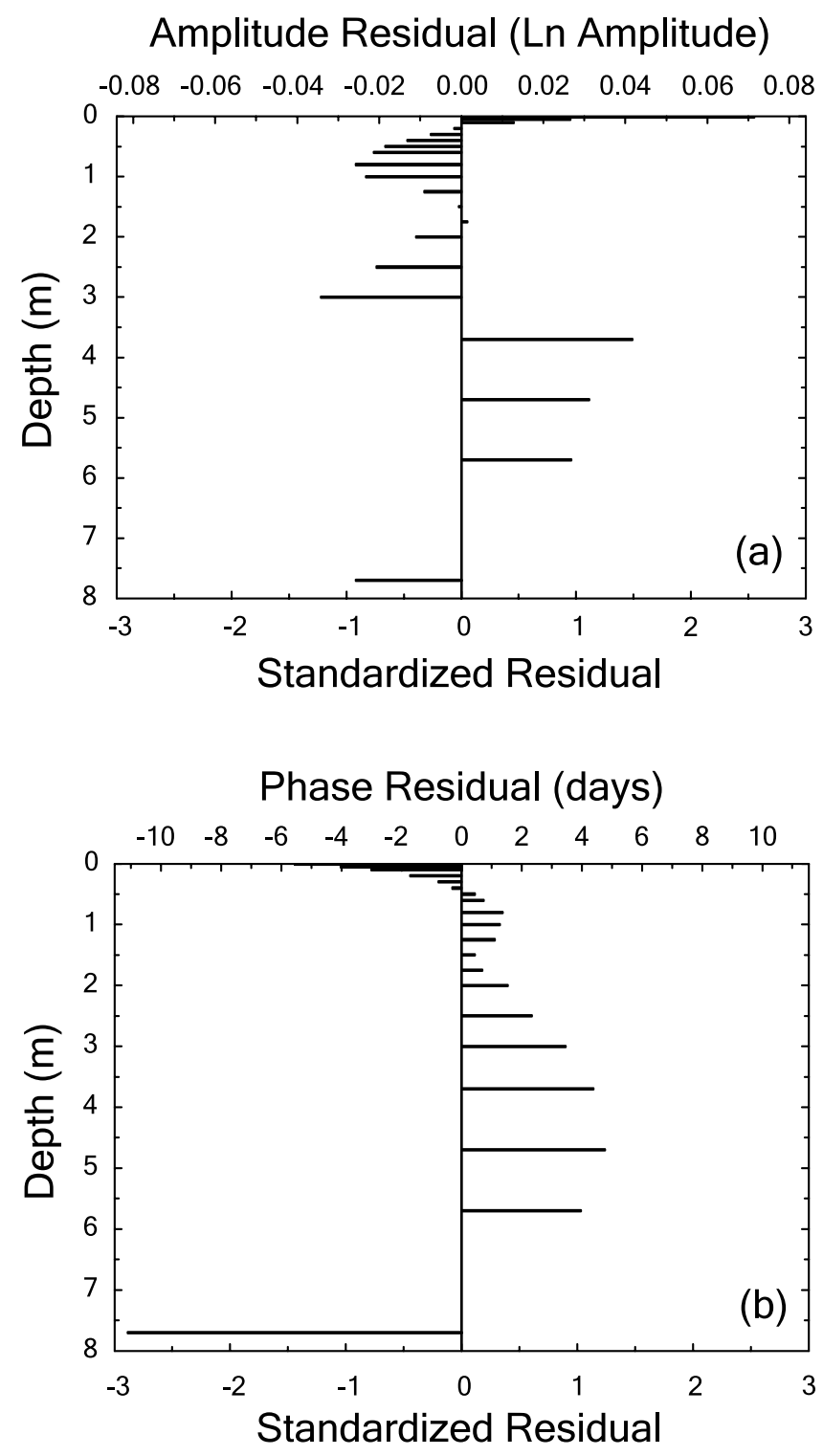

Figure 4. (a) Dimensional and standardized residuals from linear regression of the natural logarithm amplitude data versus depth. (b) Dimensional and standardized residuals from linear regression of the phase shift data versus depth. 


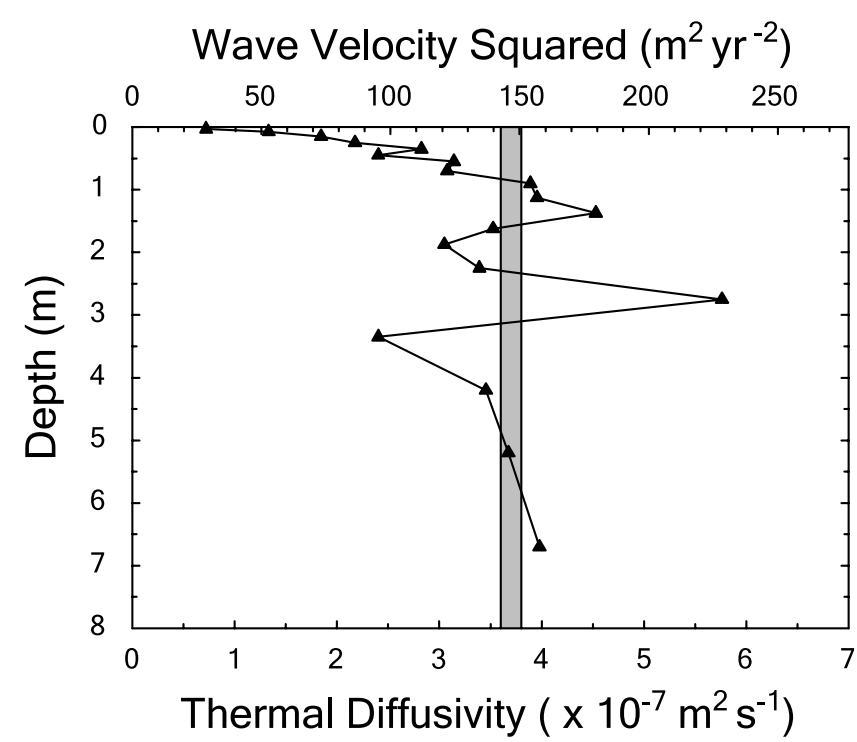

Figure 5. Interval wave velocities calculated between points of measurement (the value is squared to allow comparison to the corresponding thermal diffusivity on a linear scale). The interval thermal diffusivities were calculated from the interval wave velocities and are scaled on the bottom abscissa. The shaded region is the thermal diffusivity and wave velocity range calculated from the amplitude and phase regressions shown in Figure 3. The mean thermal diffusivity below the $1-\mathrm{m}$ depth is $3.8 \times$ $10^{-7} \mathrm{~m}^{2} \mathrm{~s}^{-1}$ with a standard deviation of $0.9 \times 10^{-7} \mathrm{~m}^{2} \mathrm{~s}^{-1}$.

point has been kept, however, to maintain a consistent depth range in both the amplitude and phase shift regressions. Excluding this point would result in a better fit and only improve the results in section 3.1.

[14] To better understand the small changes in signal propagation with depth, the interval wave velocities were calculated by dividing the depth difference by the phase shift (in time units) between measurement points. Figure 5 shows the calculated interval wave velocities at all depths, and indicates accelerating wave propagation in the upper meter of the soil. Below $1 \mathrm{~m}$ the mean wave velocity is $12.2 \mathrm{~m} \mathrm{yr}^{-1}$ with a standard deviation of $1.4 \mathrm{~m} \mathrm{yr}^{-1}$; this mean velocity compares closely to the value calculated from the regression analyses (see Table 2). The interval thermal diffusivities calculated from the interval wave velocities are also shown in Figure 5. Relatively low thermal diffusivities are apparent in the upper meter of the soil, below which the diffusivity profile converges to the narrow range of thermal diffusivities calculated from the regression analyses of the amplitude and phase data (see Table 2); the average thermal diffusivity below the $1-\mathrm{m}$ depth is $3.8 \times 10^{-7} \mathrm{~m}^{2} \mathrm{~s}^{-1}$ with a standard deviation of $0.9 \times 10^{-7} \mathrm{~m}^{2} \mathrm{~s}^{-1}$.

\subsection{SAT and GST Comparison}

[15] Extrapolation of the regression lines in Figure 3 upward to the surface $(z=0)$ allows for an estimate of the GST annual signal. The zero-depth intercepts from the regression analyses suggest a GST annual signal with an amplitude of $13.8 \pm 0.1 \mathrm{~K}$ that is phase shifted relative to the
SAT annual signal by $8 \pm 1$ days. The SAT annual amplitude determined from Fourier analysis was $17.8 \mathrm{~K}$, indicating that the GST amplitude is attenuated by $21.5 \pm 0.5 \%$ relative to the SAT. To allow comparison between the two signals on the Celsius temperature scale, we have referenced the two signals to their respective annual means. The mean annual SAT is $5.8^{\circ} \mathrm{C}$ (see section 2.2). We approximate the mean annual GST by calculating the mean at the $0.01-\mathrm{m}$ depth. Using the same method described in section 2.2, the mean annual GST was estimated to be $9.1^{\circ} \mathrm{C}$. The two signals are shown in Figure 6.

\section{Discussion}

[16] Our regression analyses of the amplitude and the phase shift of the annual signal as functions of depth confirm that conductive heat transport in a homogeneous medium explains the propagation of the annual temperature signal in the soil at Fargo with high fidelity, a result that is particularly significant given the complexity of the local climate at the site (see Figures 1 and 2). Linear functions describe the depth dependence of the natural logarithm and phase shift of the annual signal with $r^{2}$ values of 0.999 and 0.996, respectively, and give an estimate of the average thermal diffusivity of the soil of $3.7 \pm 0.1 \times 10^{-7} \mathrm{~m}^{2} \mathrm{~s}^{-1}$. This thermal diffusivity estimate closely agrees with the average thermal diffusivity of $3.8 \times 10^{-7} \mathrm{~m}^{2} \mathrm{~s}^{-1}$ calculated from results presented by Schmidt et al. [2001].

[17] The regression analyses of amplitude and phase also allow comparisons between annual GST and SAT signals. Extrapolation of the regression lines to the ground surface suggests a negligible phase shift in the GST relative to the SAT and a $21.5 \pm 0.5 \%$ attenuation of the GST amplitude relative to the SAT amplitude. Referencing the two signals to their means suggests that decoupling of the GST and SAT annual signals occurs primarily during the winter (see Figure 6). Schmidt et al.

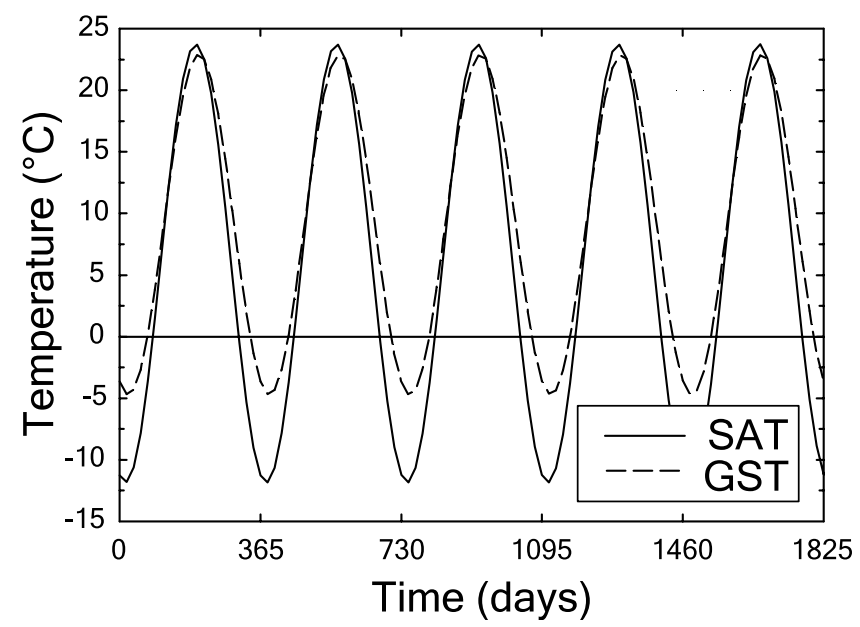

Figure 6. SAT and GST annual signals from spectral decomposition and extrapolation of amplitude and phase regression analyses, respectively. Peak-to-peak amplitudes of the SAT and GST annual signals are $35.5 \mathrm{~K}$ and $27.6 \pm$ $0.2 \mathrm{~K}$. Estimated means of $5.8^{\circ} \mathrm{C}$ and $9.1^{\circ} \mathrm{C}$ were added to the annual SAT and GST signals, respectively, to allow comparison on the Celsius temperature scale. 
[2001] also conclude that the primary nonconductive processes contributing to air and ground temperature decoupling at the Fargo site between 10 October 1980 and 16 April 1990 were related to snow cover, latent heat of freezing and spring melting.

[18] The interval thermal diffusivities shown in Figure 5 provide some insight into the net effect that winter processes can have on the thermal characteristics of the soil on seasonal timescales. While thermal diffusivity calculations over short depth intervals are subject to much greater uncertainty than the regression analyses that simultaneously address the entire data set, they provide a qualitative illustration of changing thermal characteristics in the shallow soil. The relatively low diffusivity values in the upper meter of the soil suggest that the collection of surface and nearsurface processes have a net result that can be described as a reduction of the effective thermal diffusivity of the upper soil. This observation is consistent with the dominant thermoinsulation effects of winter processes such as snow cover [Goodrich, 1982; Lewis and Wang, 1992; Gosnold et al., 1997; Zhang et al., 2001; Sokratov and Barry, 2002], the suppression of conductive heat transport through the release of latent energy during soil freezing, and zero curtain extension caused by water infiltration during snowmelt [e.g., Kane et al., 2001]. While the actual physical description of these time-dependent processes and their coupled relationships is indeed complicated, the net effect appears to be roughly equivalent to an insulation gradient in the upper meter of the soil.

\section{Conclusions}

[19] We have shown that a conductive model effectively explains the propagation of the annual signal within the soil and unequivocally supports one of the two principal assumptions of GST temperature reconstructions, i.e., that heat transport is predominantly conductive within the subsurface. We have also estimated the decoupling between GST and SAT annual signals where conductive extrapolation of subsurface temperatures has been used to reconstruct the GST signal. This has been performed at the Fargo site, where many of the cryogenic processes that may reduce the fidelity of GST and SAT tracking exist. Offsets between annual amplitudes of air and soil temperature signals ultimately are not significant for borehole climate reconstructions, so long as the difference between the two temperatures remains the same over the long term. If there are no long-term changes in the difference and the net result of cryogenic processes is to alter the thermal diffusivity of the upper meter of soil, it is unlikely that longer-period temperature changes would be significantly affected. For instance, the upper meter comprises $8.3 \%$ of the wavelength of the annual signal (assuming a thermal diffusivity of $3.7 \times 10^{-7} \mathrm{~m}^{2} \mathrm{~s}^{-1}$ ), whereas the upper meter represents only $2.6 \%$ and $0.8 \%$ of the wavelengths associated with 10 - or 100 -year cycles, respectively. It is conceivable, however, that progressive changes in annual coupling relationships between SAT and GST may generate different long-period responses in air and soil temperatures. Thus investigation of longer-period components in the two temperatures is necessary for a more complete understanding of their relationship over longer timescales. We confirm the previous conclusion of Schmidt et al. [2001] that air and soil temperatures from 0.01 to $0.8 \mathrm{~m}$ at Fargo do not contain any statistically significant long-term trends. The additional decade of data analyzed in this study also establishes the absence of long-term trends at all depths of record during the period of observation, making the Fargo site unsuitable for an investigation of longer-period coupling relationships and signal propagation. Nevertheless, our method can easily be adapted to address tracking between SAT and GST signals at other sites that display longer-period changes.

[20] Acknowledgments. We thank the senior and associate editors, Po-Yu Shen, and one anonymous reviewer for their thoughtful contributions throughout the review process. This research has been supported by NSF grant ATM-0081864, by the University of Michigan through grant OVPR4237 , and by the Department of Geological Sciences of the University of Michigan.

\section{References}

Baker, D. G., and D. L. Ruschy, The recent warming in eastern Minnesota shown by ground temperatures, Geophys. Res. Lett., 20, 371-374, 1993.

Baker, J. M., and D. G. Baker, Long-term ground heat flux and heat storage at a mid-latitude site, Clim. Change, 54, 295-303, 2002.

Beltrami, H., On the relationship between ground temperature histories and meteorological records: A report on the Pomquet station, Global Planet. Change, 29, 327-348, 2001.

Beltrami, H., Climate from borehole data: Energy fluxes and temperatures since 1500, Geophys. Res. Lett., 29(23), 2111, doi:10.1029/ 2002GL015702, 2002.

Carslaw, H. S., and J. C. Jaeger, Conduction of Heat in Solids, 2nd ed., 510 pp., Oxford Univ. Press, New York, 1959.

Goodrich, L. E., The influence of snow cover on the ground thermal regime, Can. Geotech. J., 19, 421-432, 1982.

Gosnold, W. D., P. E. Todhunter, and W. Schmidt, The borehole temperature record of climate warming in the mid-continent of North America, Global Planet. Change, 15, 33-45, 1997.

Harris, R. N., and D. S. Chapman, Mid-latitude $\left(30^{\circ}-60^{\circ} \mathrm{N}\right)$ climatic warming inferred by combining borehole temperatures with surface air temperatures, Geophys. Res. Lett., 28, 747-750, 2001.

Huang, S., H. N. Pollack, and P. Y. Shen, Temperature trends over the last five centuries reconstructed from borehole temperatures, Nature, 403, $756-758,2000$.

Kane, D. L., K. M. Hinkel, D. J. Goering, L. D. Hinzman, and S. I. Outcalt, Non-conductive heat transfer associated with frozen soils, Global Planet. Change, 29, 275-292, 2001.

Lewis, T. J., and K. Wang, Influence of terrain on bedrock temperatures, Palaeogeogr. Palaeoclimatol. Palaeoecol., 98, 87-100, 1992.

Pollack, H. N., and S. Huang, Climate reconstructions from subsurface temperatures, Annu. Rev. Earth Planet. Sci., 28, 339-365, 2000.

Putnam, S. N., and D. S. Chapman, A geothermal climate change observatory: First year results from Emigrant Pass in northwest Utah, J. Geophys. Res., 101, 21,877-21,890, 1996.

Schmidt, W. L., W. D. Gosnold, and J. W. Enz, A decade of air-ground temperature exchange from Fargo, North Dakota, Global Planet. Change, 29, 311-325, 2001.

Sokratov, S. A., and R. G. Barry, Intraseasonal variation in the thermoinsulation effect of snow cover on soil temperature and energy balance, J. Geophys. Res., 107(D10), 4093, doi:10.1029/2001JD000489, 2002.

Zhang, T., R. G. Barry, D. Gilichinsky, S. S. Bykhovets, V. A. Sorokovikov, and J. Ye, An amplified signal of climatic change in soil temperatures during the last century at Irkutsk, Russia, Clim. Change, 49, 41-76, 2001 .

J. W. Enz, Department of Soil Science, North Dakota State University, Walster Hall, Room 233, Fargo, ND 58105, USA. (john.enz@ndsu. nodak.edu)

M. J. Lewis, Michigan Center for Theoretical Physics, Physics Department, 2477 Randall Laboratory, University of Michigan, Ann Arbor, MI 48109-1120, USA. (lewism@umich.edu)

H. N. Pollack, Department of Geological Sciences, University of Michigan, 2534 C.C. Little Building, 425 E. University, Ann Arbor, MI, USA. (hpollack@umich.edu)

J. E. Smerdon, Applied Physics Program, University of Michigan, Ann Arbor, MI 48109-1063, USA. (jsmerdon@umich.edu) 